\title{
Modernización de la administración a través del empowerment, ¿es posible en las instituciones públicas?
}

Modernization of the administration through empowerment, is it possible in public institutions?

1 Samuel Alberto Pazmiño Linares Universidad de Guayaquil, Guayaquil, Ecuador samuel.pazminol@ug.edu.ec

2 Sandra Monserrate Vélez Vélez Universidad de Guayaquil, Guayaquil, Ecuador sandra.velezve@ug.edu.ec

iD https://orcid.org/0000-0003-3702-6653

https://orcid.org/0000-0001-5308-0858

Artículo de Investigación Científica y Tecnológica

Enviado: 13/12/2021

Revisado: 29/12/2021

Aceptado: 28/01/2022

Publicado:05/02/2022

DOI: https://doi.org/10.33262/ap.v4i1.1.161

administración a través del empowerment, ¿es posible en las instituciones públicas? AlfaPublicaciones, 4(1.1). https://doi.org/10.33262/ap.v4i1.1.161

ALFA PUBLICACIONES, es una Revista Multidisciplinar, Trimestral, que se publicará en soporte electrónico tiene como misión contribuir a la formación de profesionales competentes con visión humanística y crítica que sean capaces de exponer sus resultados investigativos y científicos en la misma medida que se promueva mediante su intervención cambios positivos en la sociedad. https://alfapublicaciones.com

La revista es editada por la Editorial Ciencia Digital (Editorial de prestigio registrada en la Cámara Ecuatoriana de Libro con No de Afiliación 663) www.celibro.org.ec

Esta revista está protegida bajo una licencia Creative Commons Attribution Non Commercial No Derivatives 4.0 International. Copia de la licencia: http://creativecommons.org/licenses/by-nc-nd/4.0/ 
Palabras claves: modernización, administración, empoderamiento

\section{Keywords:}

modernization, administration, empowerment

\section{Resumen}

Hoy en día las organizaciones tienen que acoplarse a la modernización, adaptándose a los cambios que se derivan de esta, para ello, deben estar en constante renovación y cambios estructurales internos, con el propósito de adecuarse a las nuevas tendencias que van naciendo. Una de estas tendencias más utilizada es la herramienta administrativa del empowerment implementada por muchas organizaciones principalmente las privadas. El objetivo de esta investigación se basa en evaluar si el empowerment como parte de la modernización de la administración es posible ser implementada en las instituciones públicas. La metodología aplicada posee un diseño bibliográfico de tipo documental. Como conclusión se menciona que la transformación de la administración se hace necesaria para conseguir las metas, efectividad y eficiencia de las organizaciones, tomando en cuenta los cambios originados producto de la modernización, tanto de tecnologías como de procesos. Cada organización es responsable de asumir los cambios que crea pertinentes para alcanzar sus objetivos y metas. Sin embargo, el empowerment resulta una herramienta muy eficaz, ya que origina que los trabajadores sean involucrados con todos los procesos dentro de las organizaciones, siendo uno de los más destacados, la participación en la toma decisiones, lo cual se traduce en un mayor sentido de pertenencia hacia su trabajo y por ende hacia la organización.

\section{Abstract}

Today, organizations have to adapt to modernization, adapting to the changes that derive from it, for this, they must be in constant renewal and internal structural changes, in order to adapt to the new trends that are being born. One of these most used trends is the Empowerment administrative tool implemented by many organizations, mainly private ones. The objective of this research is based on evaluating whether empowerment as part of the modernization of the administration is possible to be implemented in public institutions. The applied methodology has a bibliographic design of a documentary type. As a conclusion, it is mentioned that the transformation of the administration is necessary to achieve the goals, effectiveness, and efficiency of the organizations, taking into account the changes caused by modernization, both in technologies and processes. Each 
organization is responsible for assuming the changes it deems pertinent to achieve its objectives and goals. However, empowerment is a very effective tool, since it causes workers to be involved with all processes within organizations, one of the most prominent being participation in decision-making, which translates into a greater sense of belonging to their work and therefore to the organization.

\section{Introducción}

Actualmente el mundo se enfrenta a grandes cambios, los cuales afectan a las organizaciones, por ello la administración debe contar con una estructura que sea flexible y le permita ajustarse a los cambios actuales, así como también, poder pronosticar posibles escenarios que pudieran presentarse, asegurando poder hacer frente a estos.

De esta manera, uno de esos cambios que se presentan es la modernidad, la cual para Martucelli (2021), esta puede variar en función de los países o de las distintas disciplinas, esta define tanto a una sociedad como a su cultura o experiencias, también, se refiere a veces, a un periodo histórico en particular.

La modernización, es sin duda, relevante para lograr las renovaciones y reformas de cualquier organización, públicas o privadas. Ya que se trata de renovar y mejorar los procesos ya existentes, por otros, que optimicen aún más estos procesos. En lo que respecta a las instituciones públicas, se hace necesario que estas puedan modernizar sus procesos a través de nuevas tecnologías y el uso de las nuevas herramientas administrativas, que le permitan prestar sus servicios de una manera más eficaz y rápida a los ciudadanos, que son los demandantes de estos servicios. Es por ello que sea cual sea su tipo, las organizaciones deben hacer uso de la gran gama de herramientas administrativas que existen en la actualidad y adaptarse a los cambios y retos de la modernización.

En este sentido, según Pérez \& Merino (2017 citado por Mero-Vélez, 2018), La administración de empresas, está vinculada a la gestión que estás realizan, y que conforman un conjunto de preceptos, reglas y prácticas, que buscan optimizar el aprovechamiento de cada uno de los recursos de la empresa para alcanzar los objetivos.

Es por ello, que la administración está ligada a la construcción y desarrollo del sistema socioeconómico de cualquier país, por lo cual tanto las organizaciones privadas como las públicas, deben hacer uso de las herramientas administrativas existentes, y con estas lograr adaptarse a los constantes cambios y modernización de los sistemas, procesos y procedimientos, que conlleven a alcanzar los objetivos organizacionales e institucionales. 
Una de las herramientas administrativas que está siendo usada en la actualidad es el empowerment, conocido también como empoderamiento, el cual en palabras de Arroyo (2012 citado por Castro \& Núñez, 2018), el empowerment también conocido como empoderamiento, se refiere a la acción de otorgar poder y autoridad a los trabajadores en el desempeño de sus puestos de trabajo, dejándoles en claro que son ellos los responsables de su ejecución y la organización.

Asimismo, Chávez (2021), menciona que, el empoderamiento es otorgar completa autonomía y responsabilidades del control de sus respuestas en su entorno laboral, concediéndole autoridad al trabajador para que tome decisiones, lo cual tiende a aumentar la autoestima y, por ende, sentirse valorados dentro de la organización.

Dado a las definiciones consultadas, se puede mencionar que el empowerment, no es más que conferir a los trabajadores el poder de tomar decisiones y puedan contribuir a la resolución de los problemas en un momento dado, sin necesidad de que esté presente algún jefe o gerente, ya que estos le delegan esta responsabilidad. Asimismo, esto hace que los trabajadores tengan un nivel alto de participación y por ende se sientan que son importantes para la organización, contribuyendo de esta manera a que contribuyan que los objetivos o metas organizacionales sean alcanzadas.

En base a esto el objetivo de esta investigación es determinar como la modernización de la administración a través del enpowerment puede ser aplicada instituciones públicas. La metodología utilizada posee un diseño bibliográfico de tipo documental.

\section{Metodología}

La investigación se llevó a cabo bajo la metodología basada en la investigación documental, a través de la revisión bibliográfica, de documentos de carácter científico, artículos, publicaciones académicas, medios electrónicos, de diversas índoles, tomando las ideas comúnmente adoptadas por especialistas, para en un análisis descriptivo y desarrollar el tema sobre la modernización de la administración a través del empowerment.

Los procesos administrativos representan para las empresas de cualquier tipo: públicas o privadas, el uso de las herramientas administrativas, las cuales contribuyen a la mejora de sus procedimientos internos, estas tienen que ir orientadas a lograr una satisfacción del cliente o usuario, según corresponda la actividad que realicen, y por ende lograr una atención oportuna y eficaz ante los demandantes.

\section{Resultados y Discusión}

Tipos de empowerment

Empowerment Estructural 
Para Aimacaña \& Tello (2017) afirman que se refiere a las características que debe tener el ambiente de trabajo, el cual debe contar con: autonomía, carga de trabajo, apoyo a la organización y toma de decisiones. De esta forma se lograría que los trabajadores alcancen un alto nivel de empoderamiento y por ende realicen sus tareas y contribuyan a alcanzar los objetivos y metas.

En este sentido, Román et al. (2019) menciona que el empowerment:

Se concentra en las condiciones del ambiente de trabajo: variedad, autonomía, carga de trabajo, soporte de la organización y la posición en la organización, siendo estas las características que estructurales que debe contener el empleo. La existencia de estas condiciones, pueden representar un grado de satisfacción laboral, sin embargo, suelen no tomar en cuenta la percepción de los trabajadores en cuanto a esas variaciones y cambios de las condiciones ambientales.

De este modo se puede asegurar que el empowerment estructural, debe asegurar que las organizaciones cuenten con un adecuado ambiente laboral que brinde a sus trabajadores confianza, satisfacción y bienestar, así como también, otorgarles autonomía en la realización de sus labores, permitiéndoles tomar decisiones, haciéndolos participativos y necesarios para la organización, lo cual genera como resultado un alto desempeño laboral con compromiso que contribuye al logro de los objetivos planificados.

\section{Empowerment Psicológico:}

En cuanto a este tipo de empowerment, Sobrino et al. (2017) señalan que está determinado por cuatro factores:

- El significado: Es la combinación entre los valores personales del individuo y su trabajo.

- Competencia: Es el grado en que un individuo se siente capaz de llevar a cabo con las labores asignadas.

- Autodeterminación: Es el estado personal en el que un individuo se siente capaz de llevar a cabo las labores encomendadas.

- Impacto: Es el grado en que una persona puede llegar a influir en el resultado final de los objetivos de una organización.

Tal como lo señalan los diversos autores mencionados, el empowerment psicológico está orientado a mantener un alto grado de motivación de los trabajadores dentro de la organización, otorgándoles poder y autoridad para la realización de sus tareas, así como también la facultad para poder tomar decisiones.

Ventajas y Desventajas del Enpowerment 
En el trabajo de grado de Nunayalle (2021) titulado: diagnóstico del desempeño laboral y propuesta de gestión del talento humano basado en el modelo empowerment, en la corporación Portilla S.A.C. Chiclayo (2018), menciona una serie de ventajas y desventajas del empowerment que se mencionan a continuación en la tabla 1.

Tabla 1

Ventajas y desventajas del empowerment

\begin{tabular}{lll}
\hline \multicolumn{2}{c}{ Ventajas } & \multicolumn{1}{c}{ Desventajas } \\
\hline - & Un efectivo trabajo en equipo & El uso incorrecto del poder por parte de los \\
- & Alta participación de los empleados & empleados \\
- & Disminuir las tasas de rotación & La resistencia de los gerentes a delegar \\
- & Amplia el valor de la confianza en la & autoridad a un trabajador del cual no siente \\
organización & confianza o simplemente no querer hacerlo \\
$-\quad$ Disminución del ausentismo & La creencia de los gerentes en poder perder \\
$-\quad$ Alta productividad y rentabilidad & tanto su trabajo como sus privilegios dentro de \\
- Disminución de los conflictos & la organización.
\end{tabular}

Fuente: Nunayalle (2021)

Como toda herramienta administrativa, el empowerment puede representar una mejora de todos los procesos que se llevan a cabo dentro de una organización, sin embargo, la implementación de algún cambio necesita la aceptación de toda la organización, desde los niveles más altos, a los más bajos, trabajando en equipo, de esto dependerá que la puesta en marcha de esta herramienta arroje resultados positivos.

Es por ello, que los trabajadores deben estar completamente comprometidos y motivados, así como aceptar que los cambios nacen para alcanzar mejoras dentro de los procesos administrativos, los cuales favorecerán tanto a la organización como a cada trabajador que forma parte de esta.

Por ello, es importante que se reconozcan las ventajas del empowerment dentro de la organización, y entender que se orientan a la satisfacción, participación, productividad, del talento humano, quienes representan un pilar fundamental para que la aplicación de esta herramienta se logre a cabalidad.

\section{Círculo del empowerment}

De la misma manera, para Román et al. (2019) el empowerment presenta la siguiente dinámica: 


\section{Figura 1}

Circulo de empowerment

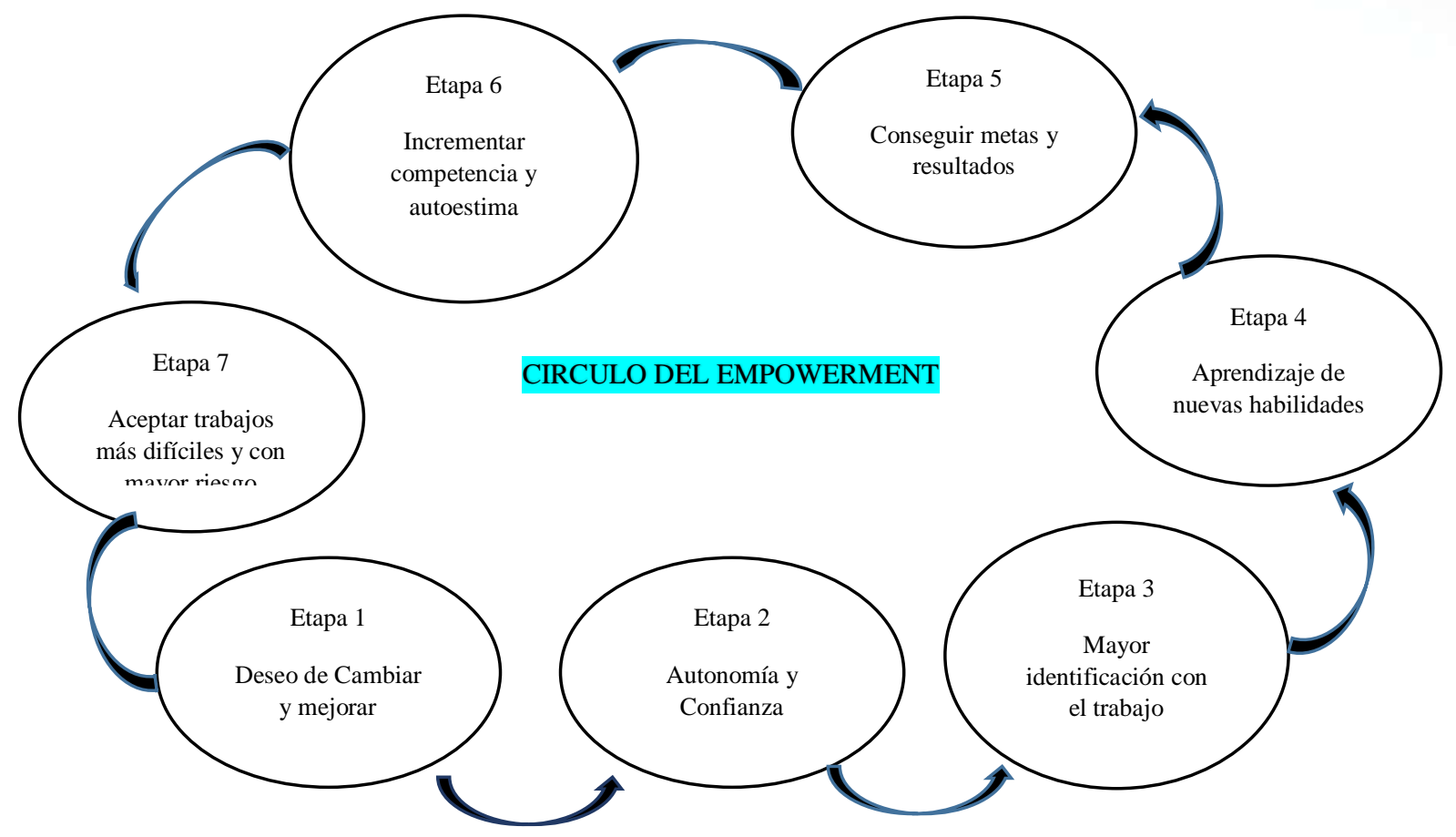

Este círculo muestra paso a paso, como debe ser implementado el empowerment dentro de las organizaciones, detallando cada una de las etapas que deben cumplirse para conseguir una exitosa implementación de esta herramienta.

\section{Dimensiones del empowerment}

Desde el punto de vista de Blanchard \& Randolph (2001 citado por Nunayalle, 2021), señala tres dimensiones que son:

\section{Compartir Información}

Para la puesta en marcha del empowerment, es importante compartir toda la información relacionada con la visión y misión organizacional, objetivos y propósitos, así como los planes de acción con todos los trabajadores, lo cual es muy importante ya que se genera confianza y ofrece a los trabajadores información relevante que les puede ayudar a tomar mejores decisiones en un momento dado.

Este proceso de compartir información contiene los siguientes pasos:

- Proporcione una formación adecuada:

- Identificar el mayor número de problemas a los que puedan enfrentarse 
- Demostrar aquellas actitudes y comportamientos que tengas mayor posibilidad de tener éxito

- Apuntan hacia fuera que nada funciona cada vez

- Celebrar cada mejora, la perfección es irrisoria.

- Usarlos como instructores de la información

- Compartir información: Para ello es necesario plantear la interrogante: ¿Qué información se necesita?

- Aliente a todo el grupo de trabajadores a apoyar y contribuir con la información

- Impedir la divulgación

- Demostrar sinceridad y franqueza

- Incentive las preguntas y desafíos

- Cuando la información requiera ser considerada cambiar su posición con habilidad.

- Hacer que todos realicen una rendición de cuentas:

- La existencia de la autoridad carente de responsabilidad puede convertirse en ingratitud.

- Cada parte del poder suele ser seductivo

- Encontrar lo que aconteció

- Preguntarse ¿Por qué ha sucedido?

- Preguntarse ¿Existía otra opción que pudo haberse utilizado?

- Depone saltar las emociones del momento.

- Visualizar los errores como oportunidades para lograr el crecimiento

\section{Autodirigir}

En el momento en que las personas maduran, se tornan autodirigidas, que no es otra cosa que las personas son capaces de diagnosticar sus necesidades, formular sus metas, habilidades y conocimientos, por ello las organizaciones deben fomentar que sus trabajadores adquieran habilidades de aprendizaje autodirigido, lo cual puede traducirse en un gran valor a largo plazo tanto para la persona como para la organización.

De esta manera, se puede decir que las dimensiones del empowerment se presentan estos tres elementos tan importantes: compartir información, autonomía y autodirigir, los cuales, al combinarse, tanto por los lideres o jefes como por los trabajadores, se pudiera implementar el empowerment de tal manera que genere los resultados deseados por la organización.

\section{Finalidad del Empowerment}

Troya et al. (2019), destacan que la finalidad del empowerment consiste en delegar poder, adoptando un modelo de horizontalidad en cuanto a las estructuras organizativas, y que 
cada miembro de esta, sienta que cuenta con apoyo y soporte, concediéndole sentido de pertenencia, poder y autoridad, con el propósito de que puedan desarrollar un liderazgo personal e integrador.

\section{Beneficios e importancia del Empowerment}

A través del empowerment se sustituyen las viejas jerarquías por equipos de trabajo autodirigidos, donde la información es compartida con todos los integrantes de la organización.

Según Suárez-Vélez (2017), señala que entre los beneficios del empowerment están dispuestos en la tabla 2

Tabla 2

\section{Beneficios e importancia del Empowerment}

\begin{tabular}{|c|c|}
\hline $\begin{array}{l}\text { Beneficios e } \\
\text { importancia }\end{array}$ & Definición \\
\hline $\begin{array}{l}\text { Distribuir } \\
\text { responsabilidad }\end{array}$ & $\begin{array}{l}\text { Se trata de repartir responsabilidades y autoridad a los trabajadores, lo cual } \\
\text { general un alto grado de compromiso por parte de estos y hace viable la } \\
\text { aplicación de una capacitación horizontal }\end{array}$ \\
\hline Liderazgo en & Representa una gran importancia la aplicación del liderazgo compartido, ya que \\
\hline Equipo & se genera por el trabajo de un equipo y no por una sola persona. \\
\hline $\begin{array}{l}\text { Forma un equipo } \\
\text { efectivo: }\end{array}$ & $\begin{array}{l}\text { Es importante que los grupos de trabajo estén compuestos de personas que sean } \\
\text { comprometidas, flexibles y creativas, así como también que sean capaces de } \\
\text { autoevaluarse en su desempeño, como también evaluar a sus compañeros, con el } \\
\text { propósito de contribuir a la obtención de información valiosa y por ende } \\
\text { gestionar la calidad. }\end{array}$ \\
\hline $\begin{array}{l}\text { Satisface y } \\
\text { cautiva al cliente }\end{array}$ & $\begin{array}{l}\text { Uno de los factores que más afectan a las organizaciones es la visión que tienen } \\
\text { sus clientes sobre ellas, siendo esta una de las razones por la que muchas veces } \\
\text { aplican cambios en su estructura. Por ello si las organizaciones desean } \\
\text { mantenerse en los mercados, no solo deben tomar en cuenta los cambios } \\
\text { tecnológicos, sino también, prestar atención a los valores y habilidades de los } \\
\text { trabajadores. }\end{array}$ \\
\hline
\end{tabular}

Fuente: Suárez-Vélez (2017)

\section{Equipos de Trabajo}

Durante el desarrollo del tema se ha nombrado constantemente los equipos de trabajo, ya que estos representan la forma de implantar el empowerment, y que en estos se centra la aplicación de este. Es por ello por lo que para De la Cruz \& Chávez (2017), los equipos 
de trabajo son: trabajadores realmente responsables de un producto o servicio, que se distribuyen el liderazgo, mejoran los procesos del trabajo, planean y toman decisiones.

Por otro lado, Romero (2020), señala que el trabajo en equipo es una estrategia, una forma o un instrumento organizacional, el cual tiene como propósito la optimización de la realización de las tareas, que conlleven a lograr las metas y objetivos planificados de la organización.

De esta forma, para la puesta en marcha del empowerment, es importante que las organizaciones diseñen los grupos de trabajo, asegurándose que puedan llevar a cabo el trabajo de forma cooperativa y en conjunto, ya que estas acciones representan al trabajo en equipo.

\section{Características del equipo de trabajo}

Según lo mencionado por Amezcua et al. (2019), el equipo de trabajo que aplica empowerment presenta las siguientes características:

- Comparten el liderazgo y las tareas administrativas

- Tienen autoridad para realizar la evaluación y la mejora de la calidad del desempeño y el proceso de información.

- Proporcionan en conjunto, ideas para la estrategia de negocios

- Están comprometidos, son flexibles y creativos

- Coordinan e intercambian tanto con otros equipos dentro de la misma organización como de otras organizaciones

\section{¿Es posible implementar el empowement en las instituciones públicas?}

No es un secreto, que las instituciones públicas son vistas como instituciones burocráticas y que mantienen procesos administrativos obsoletos, exceso de documentación, normas y procedimientos burocráticos, que originan que su funcionamiento se caracterice por prestar un mal servicio, lo cual se traduce en lentitud para dar respuestas a los requerimientos de los ciudadanos, quienes demandan estos servicios.

Esta manera de operar de las instituciones públicas, tienen que ver con las políticas del gobierno de turno, sus ideologías, siendo estos los responsables del manejo y administración de estas, usando estrategias a veces desarticuladas, tomando como base sus ideologías políticas.

Sin embargo, en la actualidad, algunos países se han preocupado por adoptar la modernización de la administración dentro de sus instituciones públicas, con el propósito de brindar un mejor servicio y una respuesta inmediata a los ciudadanos. 
El uso del empowerment, dentro de las instituciones públicas, viene a eliminar los sistemas piramidales rígidos con los que se han manejado durante mucho tiempo, eliminando la burocracia y la centralización de la toma de decisiones. Así como también asegurar que los trabajadores cuenten con las actitudes necesarias para el desempeño de los cargos, ya que generalmente, estos cargos son desempeñados por individuos que no están capacitados profesionalmente para llevarlos a cabo, lo cual origina la prestación de un mal servicio.

Según Solís \& Álava (2020), los procesos de modernización de las naciones, deben realizarse con reformas del aparato administrativo, a través de la descentralización, tanto político como administrativamente, además de llevar a cabo los procesos fundamentados en una política de construcción y/o consolidación de la democracia, asimismo suministrar a los profesionales del área administrativa, técnicas y métodos que le permitan un mejor desempeño.

Por lo antes expuesto, se puede afirmar que, si es posible implementar el empowerment, en las instituciones públicas, lo cual dependerá de la disposición de los gobiernos y de quienes estén encargados de la administración de los distintos organismos del estado.

\section{Conclusiones}

La transformación de la administración se hace necesaria para conseguir las metas, efectividad y eficiencia de las organizaciones, tomando en cuenta los cambios originados producto de la modernización, tanto de tecnologías como de procesos.

Cada organización es responsable de asumir los cambios que crea pertinentes para alcanzar sus objetivos y metas.

Sin embargo, el empowerment resulta una herramienta muy eficaz, ya que origina que los trabajadores sean involucrados con todos los procesos dentro de las organizaciones, siendo uno de los más destacados, la participación en la toma decisiones, lo cual se traduce en un mayor sentido de pertenencia hacia su trabajo y por ende hacia la organización.

También le proporciona un alto nivel de satisfacción y compromiso, dado a que a través de la aplicación de esta herramienta se les da potestad para implementar ideas, asumir riesgos, dar soluciones, haciéndolos participativos a todos los procesos de la organización.

También es importante resaltar, que las organizaciones deben garantizar una constante y permanente capacitación a los trabajadores, con el propósito de resaltar sus talentos y aprovecharlos para la consecución de los objetivos. Estas capacitaciones deben ser constantes y orientadas a desarrollar los potenciales más relevantes de cada individuo, ya 
que cada uno de estos, presentan diferentes cualidades y capacidades, para llevar a cabo un trabajo.

\section{Referencias Bibliográficas}

Aimacaña, A., \& Tello, M. (2017). Empowerment y su influencia en el compromiso organizacional en una mediana empresa: caso Industria Inplástico. 2017. Latacunga: Trabajo especial de grado de la Universidad Técnica de Cotopaxi.

Amezcua, L., Pérez, V., \& Quiroz, E. (2019). El empowerment como estrategia de crecimiento de talento humano. Ciencia Administrativa 1, 33-37.

Castro, Y, \& Núñez, V. (2018). Empowerment como una herramienta de mejora en el rendimiento laboral de los colaboradores en el área de RR. HH de la Empresa Agropucalá S.A.A., Pucalá 2017. Trabajo especial de grado de la Universidad Señor de Musipan.

Chávez, L. (2021). El empowerment como herramienta administrativa para incrementar la productividad laboral de los colaboradores de la empresa transportes Chiclayo, S.A 2019. Trabajo especial de grado de la Universidad Señor de Sipan para optar al título de Licenciado en Administración.

De la Cruz, Y., \& Chávez, F. (2017). El empowerment y la gestión administrativa en la municipalidad distrital de Palca Huancavelica- Periodo 2016. Huancavelica, Perú: Trabajo especial de grado de la Universidad Nacional de Huancavelica para optar al título de Licenciado en Administración.

Martucelli, D. (2021). Problematización de la modernidad y de la modernización de América Latina. 2021. Revista De Ciencias Sociales, 60(232), 253-274.

Mero-Vélez, J. (2018). Empresa, administración y proceso administrativo. Revista Científica FIPCAEC (Fomento De La investigación Y publicación En Ciencias Administrativas, Económicas Y Contables) 3(8), 84-102.

Nunayalle, L. (2021). Diagnostico del desempeño laboral y propuesta de gestión del talento humano basado en el modelo empowerment, en la corporación Portilla S.A.C. Chiclayo, 2018. Pimentel, Perú: Trabajo especial de grado de la Universidad Señor de Sipán para optar al título de Licenciado en Administración.

Román, J., Almeida, P., \& Ribeiro, C. (2019). Empoderamiento estructural: mejora de la capacidad innovadora de las organizaciones. Gestión y desenvolvimiento 27, 55-80. 
Romero, L. (2020). Empowerment y trabajo en equipo del personal de servicio de los establecimientos de hospedaje de tres estrellas en la ciudad de Huaraz, Región Ancash, 2018. Huaraz, Perú: Trabajo especial de grado de la Universidad Nacional Santiago Antúnez de Mayolo para optar al título de Licenciada en Turismo.

Sobrino, I., Labrador, J., Florit, M., \& Rúa, A. (2017). La dimensión evolutiva del empoderamiento psicológico y la flexibilidad psicológica en las empresas españolas: un enfoque cualitativo. Actas- Investigación cualitativa en ciencias sociales 3.

Suárez-Vélez, H. (2017). Empowermenet como estratega gerencial para mejorar la efectividad laboral. Revista Científica FIPCAEC (Fomento De La investigación Y publicación En Ciencias Administrativas, Económicas Y Contables) 2(3), 64-81.

Troya, R., Vásquez, C., \& Fajardo, L. (2019). Empowerment una herramienta estratégica como ventaja de competitividad en la administración de los gobiernos autónomos descentralizados (GADS). Revista Científica Mundo de la Investigación y el Conocimiento 3(1), 1110-1135.

\section{【 Ciencia}


El artículo que se publica es de exclusiva responsabilidad de los autores y no necesariamente reflejan el pensamiento de la Revista Alfa Publicaciones.

\section{Ciencia}

El artículo queda en propiedad de la revista y, por tanto, su publicación parcial y/o total en otro medio tiene que ser autorizado por el director de la Revista Alfa Publicaciones.
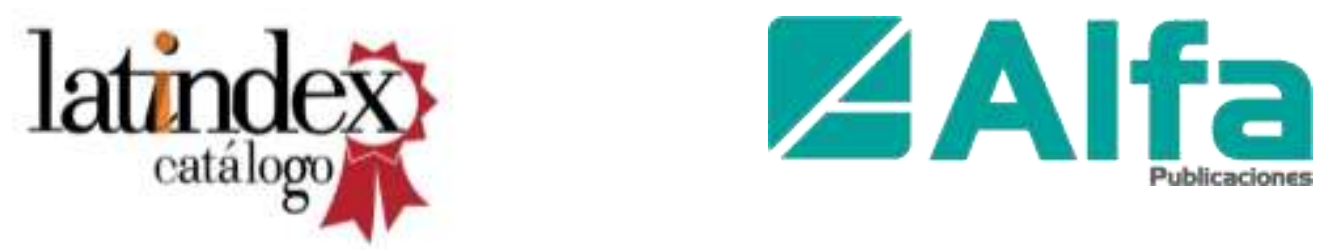

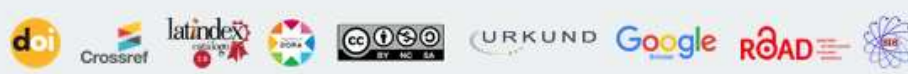
DLatinREV

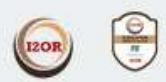

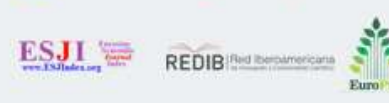

$\underbrace{}_{\text {wizdom.ai }} \mathrm{O}_{\text {OpenAIRE }}^{+}$

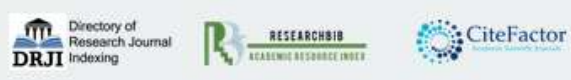

